\title{
ON THE NUMBER OF ISOLATING INTEGRALS \\ IN SYSTEMS WITH THREE DEGREES OF FREEDOM
}

\author{
CLAUDE FROESCHLE
}

Observatoire de Nice, Le Mont-Gros, 06 Nice (France)

\begin{abstract}
Dynamical systems with three degrees of freedom can be reduced to the study of a fourdimensional mapping. We consider here, as a model problem, the mapping given by the following equations:

$$
\left\{\begin{array}{l}
x_{1}=x_{0}+a_{1} \sin \left(x_{0}+y_{0}\right)+b \sin \left(x_{0}+y_{0}+z_{0}+t_{0}\right) \\
y_{1}=x_{0}+y_{0} \\
z_{1}=z_{0}+a_{2} \sin \left(z_{0}+t_{0}\right)+b \sin \left(x_{0}+y_{0}+z_{0}+t_{0}\right) \quad(\bmod 2 \pi) \\
t_{1}=z_{0}+t_{0}
\end{array}\right.
$$

We have found that as soon as $b \neq 0$, i.e. even for a very weak coupling, a dynamical system with three degrees of freedom has in general either two or zero isolating integrals (besides the usual energy integral).
\end{abstract}

\section{Introduction}

Many studies have been made in the last few years of the motion of a star in an axisymmetric galaxy or in the plane of symmetry of a spiral galaxy (see Contopoulos, 1970).

It is easy to show that this problem is completely equivalent to the study of a dynamical system with two degrees of freedom. The present paper deals with the motion of a star in a galaxy without any symmetry, i.e. the study of a dynamical system with three degrees of freedom.

One of the most fruitful methods in the case of two degrees of freedom has been the method of 'Surface of Section'. This method dates back to Poincaré (1892) and consists essentially in considering not a complete trajectory in the phase space but only its successive intersections with a certain 'Surface of Section'.

Let us consider, quite generally, a conservative system with $n$ degrees of freedom. The corresponding phase space has $2 n$ dimensions. However, a given trajectory must lie on a manifold with $2 n-1$ dimensions corresponding to a given value of the energy. In this manifold we define a 'surface of section' which is a given sub-manifold with $2 n-2$ dimensions and we consider the successive intersections of the trajectory with this sub-manifold.

For $n=2$ the 'surface of section' has two dimensions and the corresponding mapping $T$ which maps an intersecting point into the next one is an area preserving mapping. In particular it is easy to know whether an isolating integral does exist. In this case the set of points obtained by repeated application of the mapping seems to lie exactly on a closed curve. On the other hand it has been found that direct study of given area preserving mappings displays the usual features of dynamical systems with two degrees of freedom.

For $n=3$ the surface of section has four dimensions and the problem of finding 
whether one or two other isolating integrals exist in addition to the integral of the energy is more difficult than for $n=2$. Nevertheless, using various numerical methods we have studied such systems taking the three-dimensional restricted problem as an example. We have found (Froeschlé, 1969, 1970) that for orbits close to one of the primaries the points appear to lie on a two-dimensional manifold and therefore two isolating integrals seem to exist besides the Jacobi integral. More distant orbits appear to fill a manifold with four dimensions and therefore the two isolating integrals have disappeared. In fact they appear to vanish at the same time.

In order to study the number of isolating integrals in dynamical systems with three degrees of freedom or more exactly to find whether cases of transition with only one isolating integral besides the integral of the energy do exist, we have taken a four-dimensional mapping $T$ of $R^{4}$ into itself as a model problem. We study the set of points obtained by repeated applications of the mapping $T$, i.e.

$$
P_{0}, P_{1}=T\left(P_{0}\right), \ldots, P_{n}=T^{n}\left(P_{0}\right)=T\left(P_{n-1}\right) \text {. }
$$

In Section 2 we give some properties of the mapping $T$ and its features when the coupling is equal to zero.

In Section 3 we study the number of isolating integrals for the coupled case using slice-cutting methods.

\section{The Mapping}

We take a mapping $T$ of the (xyzt) space over itself defined by

$$
T\left\{\begin{array}{l}
x_{1}=x_{0}+a_{1} \sin \left(x_{0}+y_{0}\right)+b \sin \left(x_{0}+y_{0}+z_{0}+t_{0}\right) \\
y_{1}=x_{0}+y_{0} \\
z_{1}=z_{0}+a_{2} \sin \left(z_{0}+t_{0}\right)+b \sin \left(x_{0}+y_{0}+z_{0}+t_{0}\right) \\
t_{1}=z_{0}+t_{0}
\end{array}\right.
$$

The determinant of the Jacobian matrix is equal to 1. This mapping has been suggested by Arnold (1965).

If $b=0$ then the mapping $T$ is the product of two area-preserving mappings $T_{1}$ of $(x, y)$ on itself and $T_{2}$ of $(z, t)$ on itself. These mappings are inverse mappings of those given by Taylor (1969). Each of these mappings displays the well-known features of problems with two degrees of freedom (Hénon, 1969).

Figure 1 shows typical sets of points for the transformation $T_{1}$, i.e.

$$
T_{1}\left\{\begin{array}{l}
x_{1}=x_{0}+a_{1} \sin \left(x_{0}+y_{0}\right) \\
y_{1}=x_{0}+y_{0}
\end{array} \quad(\bmod 2 \pi)\right.
$$

for some initial conditions of $x_{0}$ and $y_{0}$ given in Table $\mathrm{I}$ and for $a_{1}=-1.3, N$ being the total number of points plotted for each set of points.

For certain values of the initial conditions $x_{0}$ and $y_{0}$ the points $\left(x_{n}, y_{n}\right)$ generated by $T_{1}$ seem to lie on an invariant curve; this indicates the invariance of one isolating integral. 
The figure shows clearly two curves. This seems to indicate that the points $P_{n}$ lie on a two-dimensional manifold with two sheets.

If $x_{0} y_{0} z_{0} t_{0}$ are such that an invariant curve exists for $T_{1}$ but not for $T_{2}$, then one isolating integral exists in the uncoupled case $b=0$. What can one expect in this case for $b \neq 0$ ? In order to study this case of transition the points $P_{n}$ for which $\left|z_{n}-z_{0}\right|<$ $\varepsilon_{2}$ have been plotted for various values of $N$, the total number of points. The condition $\left|z_{n}-z_{0}\right|<\varepsilon_{2}$ is no longer a section but it reduces the number of plotted points to manageable propositions.

Figure 3 summarizes the results of these experiments for $N=20000$ (20000) 120000 . For each case we have the initial conditions $x_{0}=0.5, y_{0}=0.5, z_{0}=0.5, t_{0}=3$ and $b=0.01, \varepsilon_{2}=0.1$.

For $N$ less than 80000 , the points lie on strips including the invariant curve which corresponds to the zero coupling case. The width of these strips increases with $N$. For $N$ greater than 80000 the points are scattered and ergodicity appears.

In order to follow the phenomenon in more detail we have used slice cuttings on $n$ only (same initial conditions and same parameters). Hence we have plotted the projections on the $(x, y)$ plane of the points $P_{n}$ for which $N<n<N+100$ with $N=10000$ (10000) 200000.

Let us come back to Figure 1 which shows that with some given initial conditions the points lie either on an invariant curve or are scattered in the ergodic zone. On the other hand, Figure 4 shows the two types of behaviour. The curve begins wandering in a quasi-random fashion; it changes into islands $(N=70000)$, becomes a

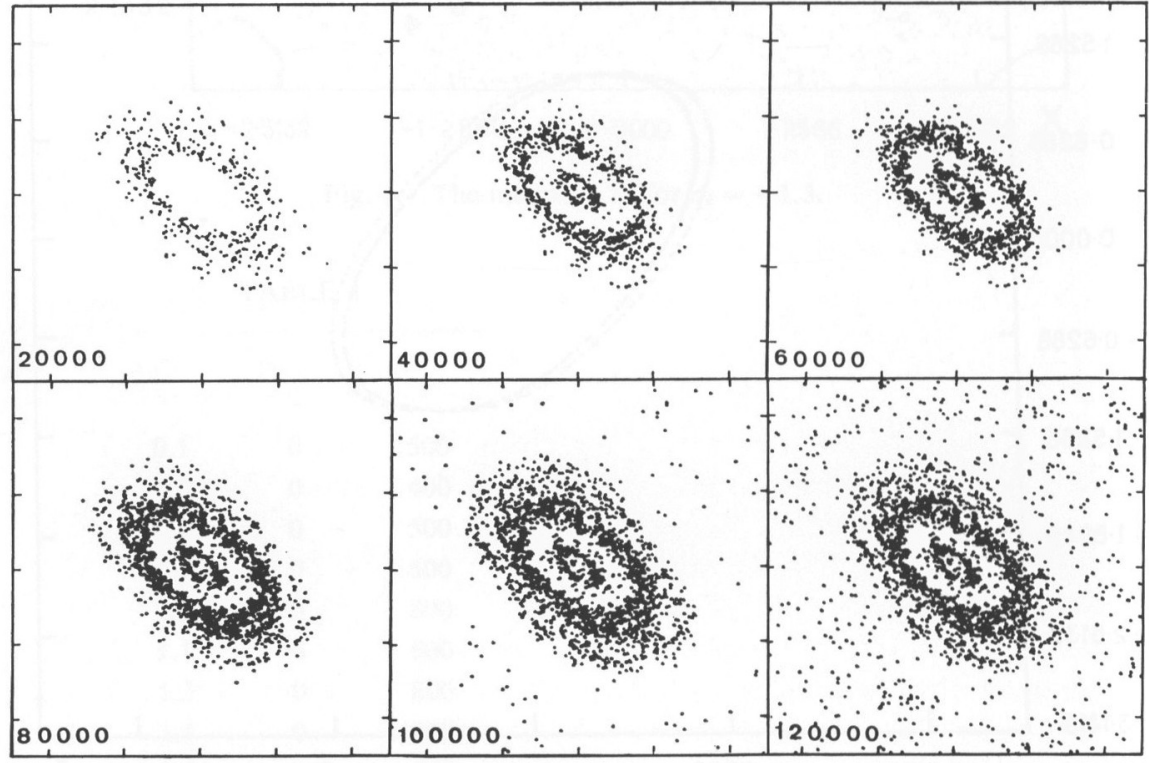

Fig. 3. Projections on the $(x, y)$ plane of the set of points $P_{n}=T\left(P_{n-1}\right), n=0, N-1$ for the initial conditions $x_{0}=0.5, y_{0}=0.5, z=0.5, t_{0}=3$ and $a_{1}=-1.3, a_{2}=-1.3, b=0.01$ when $z_{n}-z_{0}=0.1$ and $N=20000$ (20000) 120000 . 


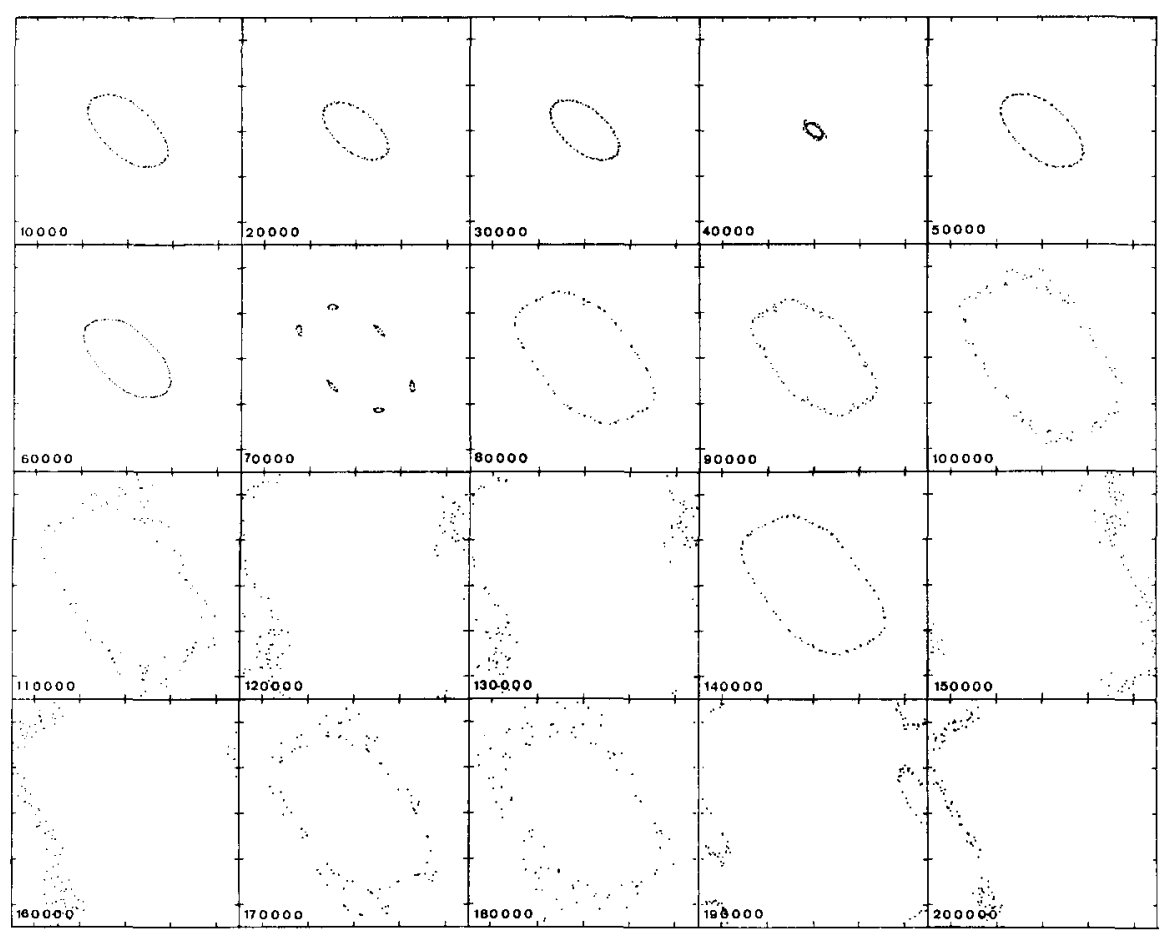

Fig. 4. Projections on the $(x, y)$ plane of the set of points $P_{n}=T\left(P_{n-1}\right) n=0,-, 110000$ for the initial conditions $x_{0}=0.5, y_{0}=0.5, z_{0}=0.5, t_{0}=3$, and $a_{1}=-1.3, a_{2}=-1.3, b=0.01$ when $N<n \leqslant N+100$ with $N=10000(10000) 200000$.

curve again, then becomes ergodic $(N=110000$ to 130000) and reverts to a curve $(N=140000)$, becomes ergodic again $(N=150000$ to 200000$)$. The following explanation suggests itself: since $T_{2}$ has no isolating integral, the points $\left(z_{n} t_{n}\right)$ behave in an ergodic, quasi-random fashion. Therefore the coupling term $b \sin \left(x_{0}+y_{0}+z_{0}+t_{0}\right)$ produces a quasi-random perturbation of the points $\left(x_{n} y_{n}\right)$. As a result the value of the former isolating integral of $T_{1}$ is subjected to a kind of random walk.

In order to have some quantitative information about this random walk, a measure $D_{j}$ of the dimension of the curve has been computed where

$$
D_{j}=\left(\sum_{n=(j-1) 100}^{n=j \times 100}\left(x_{n}^{2}-a_{1}\left(y_{n}^{2}+x_{n} y_{n}\right)\right)\right) / 100 .
$$

The quadratic term in this expression is constant in the vicinity of the origin in the linear approximation.

Figure 5 shows the variations of $D_{j}$ with $j$ for different cases. The lowest line shows the results for the integrable case (two isolating integrals exist) for which $D_{j}$ is approximately constant. On the other hand the upper line shows the variations of $D_{j}$ when only one isolating integral exists initially. The variations of $D_{j}$ are quite 


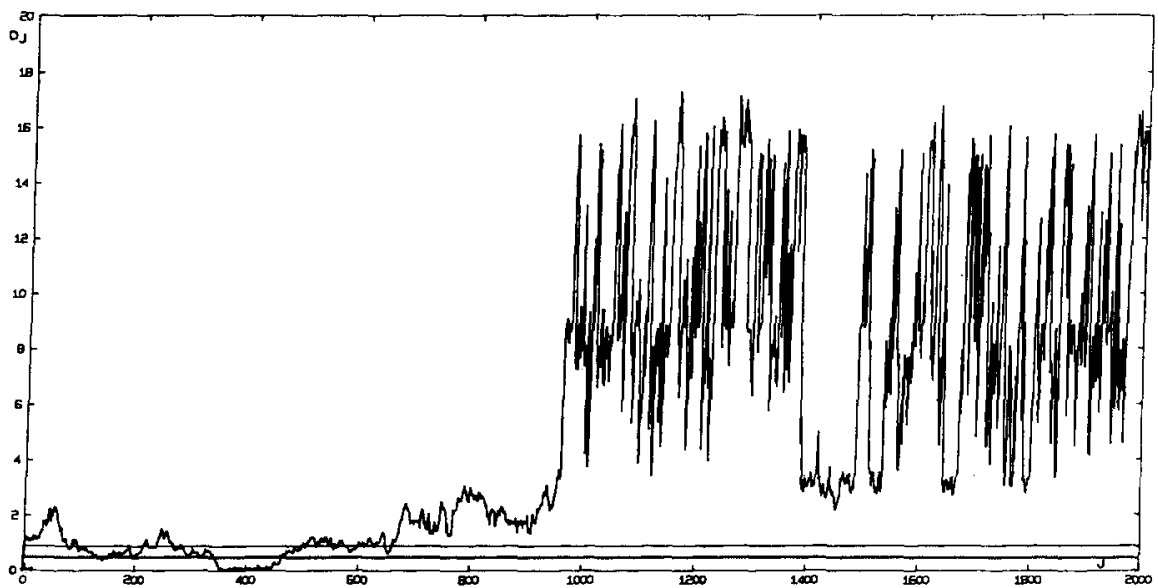

Fig. 5. $D_{f}$, a measure of the dimension of the curve, against $\mathbf{J}$ for different characteristic cases. upper line for the case of Figure 4. - intermediate line for the case of Figure 4 but with $b=0$. lowest line for the case of Figure 2.

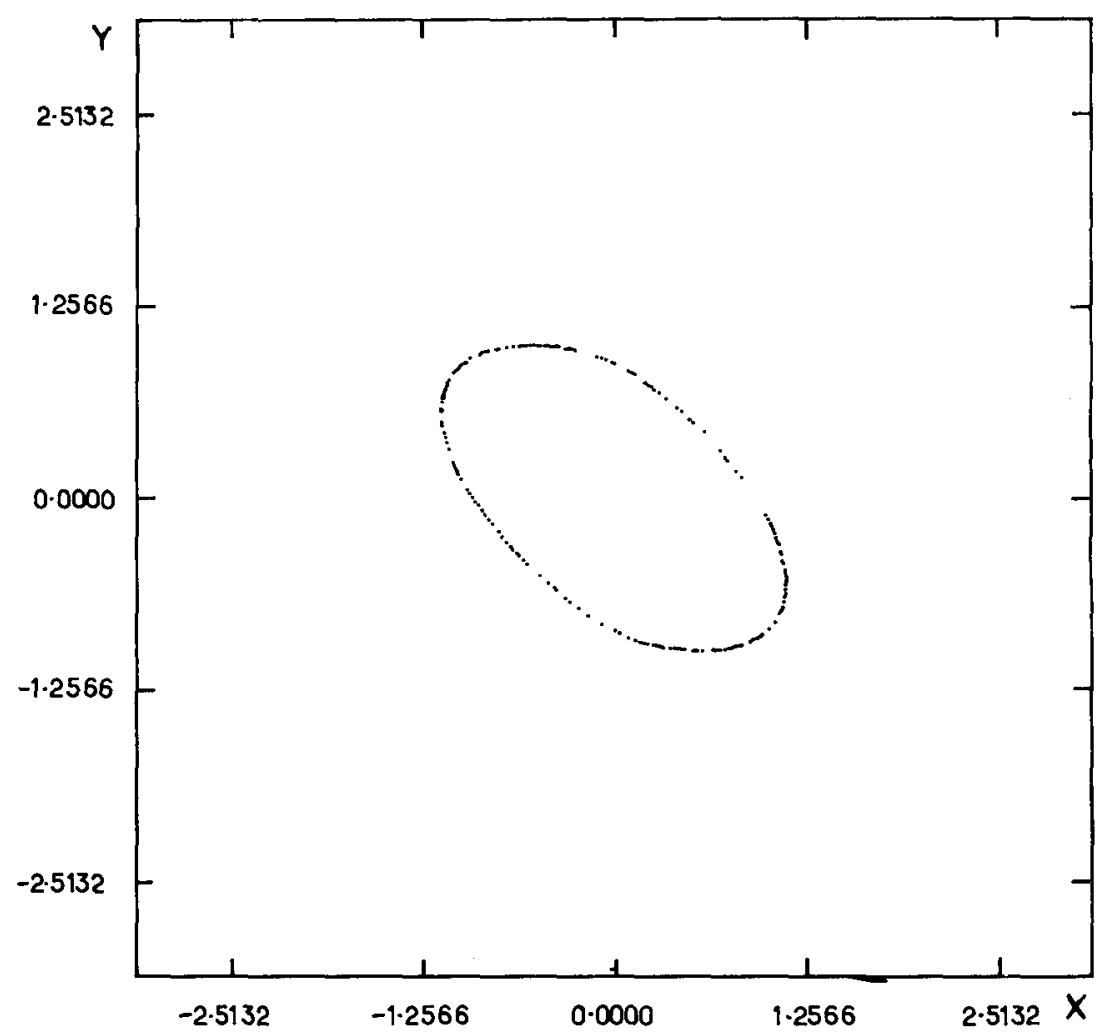

Fig. 6. Projection on the $(x, y)$ plane of the different sections, for the case of Figure 4 but with $b=0$. 
large and are in good agreement with the graphical results given by Figure 3 and Figure 4.

Figure 6 shows the same results as Figure 4 for $b=0$, where all the sections in $n$ have been plotted on the same figure. It shows clearly that the effects of the rounding errors of the computer are negligible although they could have produced the same effects as the coupling. This is also shown in Figure 5 where the intermediate line, shows clearly that $D_{j}$ remains constant with $j$.

\section{Conclusion}

The results found with the help of a four-dimensional mapping suggest that, apart from particular cases (such as $b=0$ in the present model), a dynamical system with three degrees of freedom has in general either two or zero isolating integrals (besides the usual energy integral). The disappearance of one of the two isolating integrals entails the disappearance of the other.

This agrees with results obtained earlier for a particular case of the three-dimensional restricted problem (Froeschlé, 1970). A similar effect probably exists in systems with more than three degrees of freedom, i.e. a dynamical system with $n$ degrees of freedom has in general either $n-1$ or zero isolating integrals (besides the usual energy integral).

\section{Acknowledgement}

It is pleasure to thank $\operatorname{Dr}$ M. Hénon for his constant guidance and encouragement, throughout this research.

\section{References}

Arnold, V. I.: private communication to Dr M. Hénon.

Contopoulos, G.: 1970, Cont. from the Ast. Dept. Univ. of Thessaloniki, No. 53.

Froeschle, C.: 1970, Astr. Astrophys. 4, 115.

Froeschle, C.: 1970, Astr. Astrophys. 5, 177.

Hénon, M.: 1969, Qlıart. Appl. Math. 27, 291.

Poincaré, H.: 1892, Les Méthodes Nouvelles de la Mécanique Céleste, Gauthier-Villars, Paris.

Taylor, J. B.: 1969, private communication; see Culham Laboratory Progress Report CLM-PR-12. 